\title{
Theoretical study of nonlinear optical properties of some azoic dyes
}

\author{
Djebar Hadji * and Ali Rahmouni \\ Laboratoire de Modélisation et Méthodes de Calcul, Faculté des sciences, Université de Saïda, Algérie.
}

\begin{abstract}
In this paper we presented semi-empirical PM3, ab-initio (HF, MP2) and DFT (B3LYP, B3PW91) calculation of the dipole moment, polarizability, and first hyperpolarizability of some azoics dyes derivatives which have electron donor and electron acceptor groups on either sides. The first hyperpolarizability of these molecules was calculated with PM3 method, HF/6-31G**, HF/6-31+G**, HF/6-31++G**, DFT with both B3LYP and B3PW91 functional and MP2/6-31++G** based on finite field approach using GAUSSIAN03 program. The effects of the intramolecular charge transfer (ICT) from the donor to the acceptor groups on the molecular geometry and atomic charge distribution of these NLO (nonlinear optical) chromophores are derived from the corresponding HF, MP2 and DFT calculations. The variation of these properties have also been correlated to E (HOMO-LUMO) gap and to the nature of the highest occupied molecular orbital HOMO and the lowest unoccupied molecular orbital HOMO. This study reveals that the azoics dyes derivatives have large hyperpolarizability values; hence they may be used in the development of nonlinear optical materials.
\end{abstract}

Keywords: semi-empiric; ab initio; DFT; hyperpolarizability; HOMO; LUMO; Intramolecular charge transfer.

\section{Introduction}

With appropriate electron donor/acceptor ring substitution like azobenzene molecules, the $\pi$ electron delocalization of the extended aromatic structure can yield high optical nonlinearity, so azo chromophores have been the subject of extensive study for nonlinear optical applications ${ }^{1,2}$. These chromophores are versatile molecules and have received much attention in both fundamental and applied research. The nonlinear optics (NLO) of materials was started after Kerr's observation that quadratic electric field induced a change in the refraction index, known as the Kerr effect ${ }^{3}$ in 1875. This was followed by the observation of the Pockel effect ${ }^{3}$ in 1883. The limited use of this nonlinear effect was completely changed after the invention of the laser by Maiman 4 in 1960 . This was followed by the observation of second harmonic generation (SHG) in quartz ${ }^{5}$ in 1961. The NLO developed extensively throughout this period. Molecules with large optical nonlinearity have become the focus of current research in view of their potential applications in various photonic technologies, including all-optical switching and data processing ${ }^{6-}$ 11. Three classes of NLO materials were identified namely multi-layered semi-conductor structures, molecular based macroscopic assemblies and traditional inorganic solids. A variety of inorganic, organic and organometallic molecular systems have been studied for NLO activity ${ }^{12}$. Organic molecules, in particular exhibiting extended $\pi$-conjugation,

*Corresponding author:Djebar Hadji

E-mail address: hadji120780@yahoo.fr

DOI: http://dx.doi.org/10.13171/mjc.4.4.2015.15.07.22.50/hadji show enhanced second order NLO properties. Recent results ${ }^{13}$ also suggest that molecular based macroscopic $\pi$-electron systems possess many attractive NLO characteristics. Compared to the traditional inorganic solids, these organic materials show ultra-fast response times, lower dielectric constants, better process ability characteristics and enhanced NLO responses. Since the molecular chromophores are responsible for the NLO activity of the molecule based materials, the search for novel molecules capable of manipulating electric fields, especially photonic signals, is currently an intense area of research. Prasad and Williams ${ }^{11}$ explained that certain classes of organic materials exhibit extremely larger NLO and electro optic effect. However, in the past two decades, after the development of quantum methods, the science of chromophores design has taken a different route especially due to the birth of quantum chemistry packages. A large number of publications reporting new NLO response formalisms appeared in the recent literature ${ }^{14-18}$.

The subject of this work is to design a range of these molecular systems showing NLO activity. The approach is based on the concept of intramolecular charge transfer (ICT) between donor and acceptor through an azoics dyes moiety. In this research work, dipole moment $\mu$, polarizability $\alpha$, and first hyperpolarizability $\beta$ are calculated using semiempirical PM3, ab initio and DFT method using GAUSSIAN03 program [19] for acrylics dyes derivatives. The designing of systems with high intra 
CT between donor and acceptor will lead to a very large value of $\beta$.

\section{Azobenzene systems}

Apart from NLO properties, an azobenzene system exhibits photo-switching properties, which make it suitable for variable kind of applications. Azobenzenes exist in two isomeric forms, the $\mathrm{E}$ (Trans) and $\mathrm{Z}$ (Cis) form, which can be recognized by differences in their UV absorption spectra ${ }^{26,27}$. In principle, azobenzenes can function as molecular switches by applying light of different wavelengths to obtain varying amounts of Cis and Trans isomers, where the excess of one of the two isomers may be detected by a change in UV absorption spectra. Photo-anisotropic materials are widely investigated for optical storage and processing applications. Smart materials are a branch of science that uses, particularly in biomedical sciences, this particular behavior of azobenzene molecules to switch with a long-lived cis-state in order to photocontrol the enzyme activity of a histone deacetylase-like amidohydrolase [28].

The most promising of these materials seem to be azo-dye-polymer systems because of their large value of photo-induced birefringence. Azobenzene systems possess advantages for high optical nonlinearities due to photo-induced trans-cis isomerization, molecular reorientation and nonlinear absorption but, the micro-crystal which causes light diffusion is not suitable for a quality optical surface. Among the potential materials for reversible optical data storage, liquid crystalline azobenzene side-chain polymers are especially promising due to their unique optical properties. Holograms of very high diffraction efficiency and exceptional stability can be written (and erased) by laser illumination in this material. Azobenzene polymers are interesting because they combine the properties of anisotropy with photo-responsive behavior that give rise to applications in areas such as LC displays, NLO materials and information storage devices. Etc ... [28-29].

Structures of the studied molecules

The azoics dyes used in this work are sketched in Figure 1.

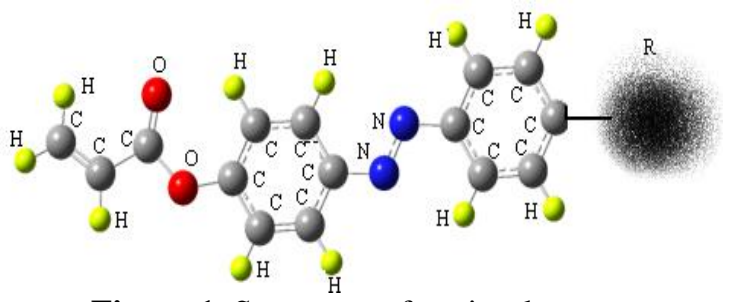

Figure 1. Structures of azoics dyes

Table 1. The structures of the studied molecules

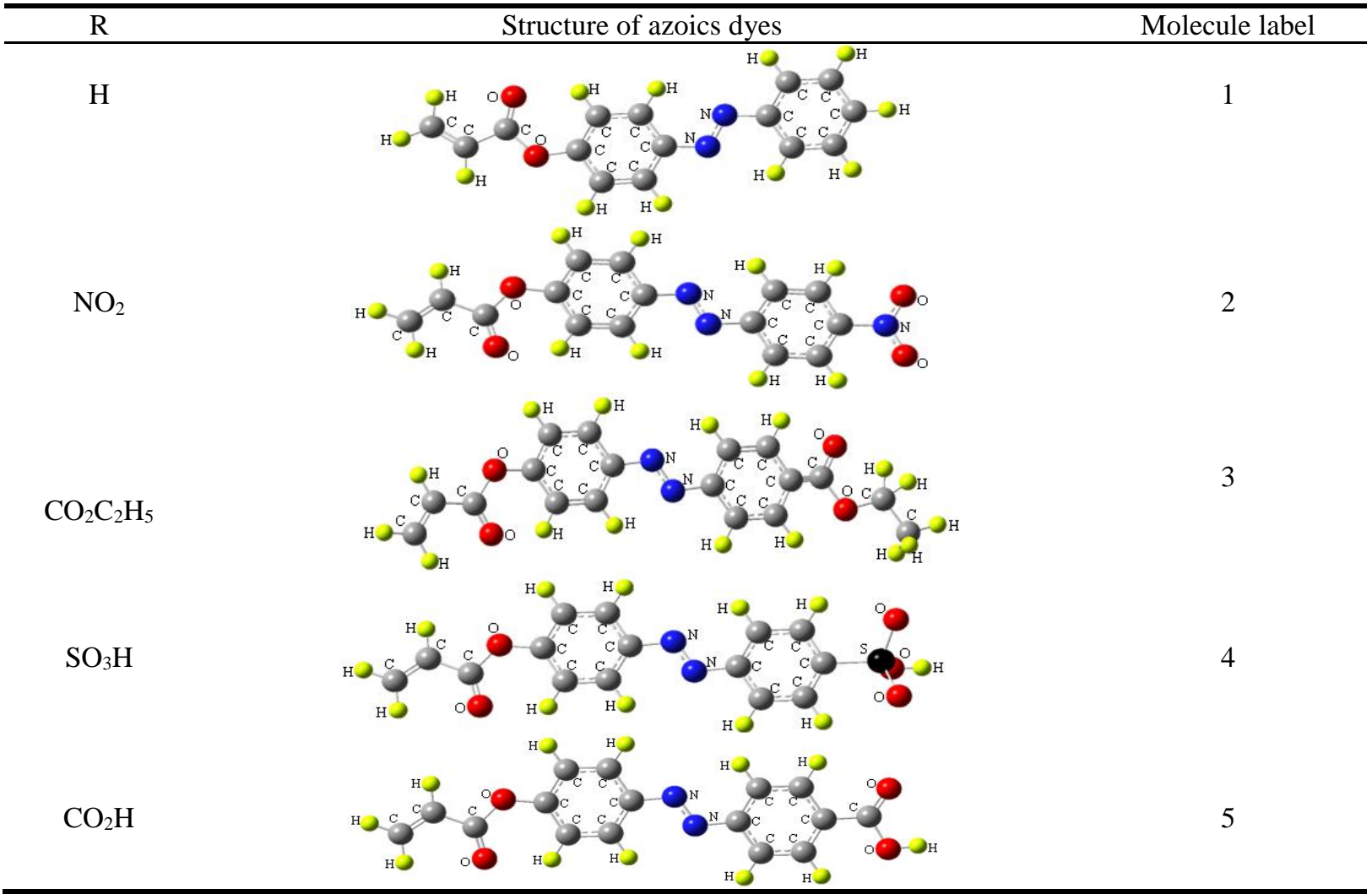

These molecules were synthesized in two steps. The first one is azophenol synthesis.by a diazotization reaction followed by an electrophyl substitution with a phenol.

\section{Principe of nonlinear optic}

Nonlinear optics deals with the interactions of strong electromagnetic fields in various media to produce new fields altered in phase, frequency, 
amplitude or other propagation characteristics from the incident fields. When a beam of light is impinged into a material, it causes the charges of the atoms to oscillate. In the linear regime the amount of charge displacement is proportional to the instantaneous magnitude of the electric field. The charges oscillate at the same frequency of the incident light. The oscillating charges either radiate light at that frequency or the energy is transferred into nonradioactive modes that result in material heating or other energy transfer mechanisms. With small fields, the displacement of charge from the equilibrium position, polarization $(P)$ will be proportional to the applied field, $E$ :

$$
p=p_{0}+\mu_{i j}^{(1)} E_{j}
$$

With sufficiently intense laser radiation the relation may not be valid, and must be generalized to nonlinear polarization

$$
p=p_{0}+\chi_{i j}^{(1)} E_{j}+\chi_{i j k}^{(2)} E_{j} E_{k}+\chi_{i j k l}^{(3)} E_{j} E_{k} E_{l}+\ldots
$$

$P_{0}$ Static dipole moment $\chi^{(n)}$ nth order of susceptibility

At the molecular level the nonlinear polarization $p$ is

$$
p=\mu_{i}^{0}+\alpha_{i j} E_{j}+\beta_{i j k} E_{j} E_{k}+\gamma_{i j k l} E_{j} E_{k} E_{l}+\ldots
$$

With $\mu_{i}^{0}$ molecular dipole moment, $\alpha_{i j}$ the linear polarizability, $\beta_{i j k}$ the first hyperpolarizability and $\chi_{i j k l}$ the second hyperpolarizability.

\section{Computational details and methods}

All calculations were made in gas phase using GAUSSIAN03 [19] set of programs. The geometry optimization was performed in DFT/B3LYP/6$31 \mathrm{G}^{* *}$. All geometrical parameters were allowed to vary independently apart from planarity of the rings. NLO calculations were performed at PM3, HF/6$31 \mathrm{G}^{* *}, \mathrm{HF} / 6-31+\mathrm{G}^{* *}, \mathrm{HF} / 6-31++\mathrm{G}^{* *}, \mathrm{MP} 2 / 6-$ $31++\mathrm{G}^{* *}$ and DFT (B3LYP, B3PW91) using 6$31++\mathrm{G}^{* *}$ basis set which has been found to be adequate to obtain reliable trends in $\beta$ values [20-21]. After optimizing the geometry, the $\beta_{\mathrm{ijk}}$ tensorial components are determined using Finite-Field theory (FF) implemented in the same GAUSSIAN03 package. All the molecules have a conjugated backbone made up of azobenzene, with a fixed acceptor group, which is a nitro group, $\mathrm{NO}_{2}$ and $\mathrm{HSO}_{3}$, or donor group which is $\mathrm{COOC}_{2} \mathrm{H}_{5}$. The |HOMO-LUMO| energetic gap was calculated using
PM3 method for the molecules 3 and 5 in various geometries.

\section{Results and Discussion}

The molecules studied in this investigation employing semi-empirical, ab initio and DFT approaches, are shown in Table 1. The study involves the calculation of dipole moment, polarizability, and first hyperpolarizability for all five derivatives. It is intended to compare the electronic effect on the first hyperpolarizability of azoics dyes derivatives upon substitution of donor and acceptor groups at appropriate positions in the azoics dyes moiety.

First hyperpolarizability is a third rank tensor that can be described by a $3 \times 3 \times 3$ matrix. The 27 components of the $3 \mathrm{D}$ matrix can be reduced to 10 components due to the Kleinman symmetry [22] $\left(\beta_{x y y}=\beta_{y x y}=\beta_{y y x}=\beta_{y y z}=\beta_{y z y}=\beta_{z y z \ldots} \quad\right.$ likewise other permutations also take same value). In the lower tetrahedral format, the GAUSSIAN03 output file provides 10 components of this matrix as $\beta_{x x x}, \beta_{x x y}$, $\beta_{x y y}, \beta_{y y y}, \beta_{x x z}, \beta_{x y z}, \beta_{y y z}, \beta_{x z z}, \beta_{y z z}, \beta_{z z z}$. Many types of hyperpolarizability have been discussed in the literature denoted as $\beta_{\text {vec }}(\beta$ vector), $\beta \|$ ( $\beta$ parallel), and $\beta_{\text {tot }}\left(\beta\right.$ total). $\beta_{\text {vec }}$ which is the component along the dipole moment direction can be measured experimentally using stark spectroscopy [23]. However, the theoretical chemists are concerned with $\beta \|$, which is the component parallel to the ground state CT direction, and the other is the total hyperpolarizability $\beta_{\text {tot. In this investigation, we }}$ report $\beta_{\text {tot }}$ for all the molecules listed in Table 1 . The components of $\beta$ can be calculated using the following equation:

$$
\beta_{i}=\beta_{i i i}+\frac{1}{3} \sum_{i \neq j}\left(\beta_{i j j}+\beta_{j i j}+\beta_{j j i}\right)
$$

Using the $\mathrm{x}, \mathrm{y}$ and $\mathrm{z}$ components of $\beta$, the magnitude of the first hyperpolarizability tensor can be calculated.

$$
\beta_{t o t}=\left(\beta_{x}^{2}+\beta_{y}^{2}+\beta_{z}^{2}\right)^{1 / 2}
$$

The complete equation for calculating the magnitude of $\beta$ from GAUSSIAN03 output is given as follows:

$\beta_{t o t}=\left[\left(\beta_{x x}+\beta_{x y y}+\beta_{x z z}\right)^{2}+\left(\beta_{y y y}+\beta_{y z z}+\beta_{y x x}\right)^{2}+\left(\beta_{z z z}+\beta_{z x}+\beta_{z y y}\right)^{2}\right]^{1 / 2}$

Since the first hyperpolarizability tensors values of the GAUSSIAN03 program output file are reported in atomic units (au), we reported $\mu, \alpha$ and $\beta$ in au: 
Table 2: Dipole moment (D), polarizability (au) and first hyperpolarizability (au) of azoics dyes

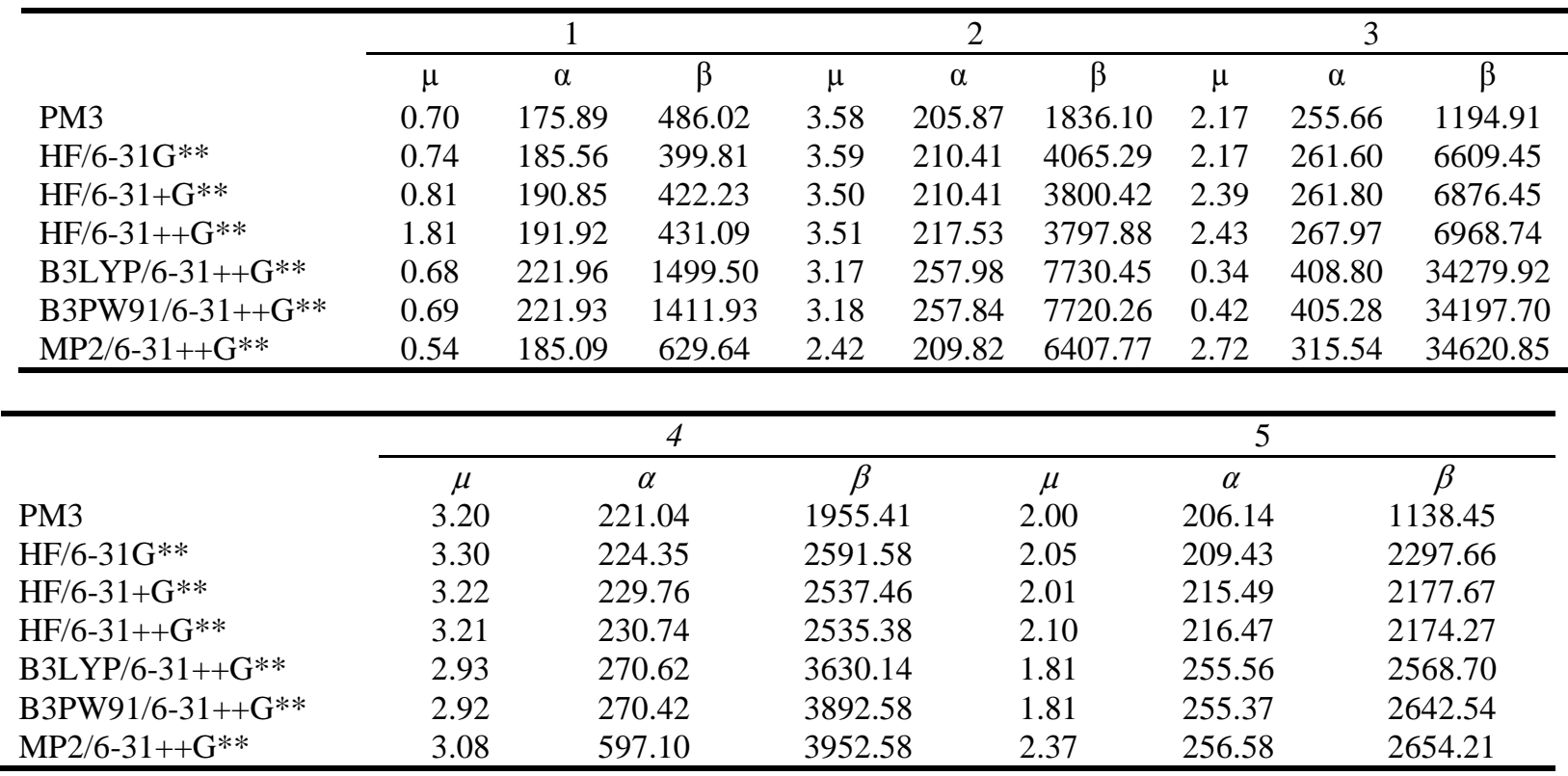

The semi-empirical level calculation is used in the Gaussian software package; in particular polarizability could be calculated using MNDO, AM1, and PM3 Hamiltonians. The critical question surrounding such an approximation, as oppose to the $a b$ initio algorithm is the reliability of results. The PM3 method [24] was also employed to determine the dipole moment $\mu$, polarizability $\alpha$, and first hyperpolarizability $\beta$ for the molecules given in Table 1. The aim of this part of the calculation was to compare the ab initio and DFT calculations, with the semi-empirical level. It was found that the semiempirical value of $\beta$ is $100 \%$ increased respect to the $\mathrm{HF} / 6-31 \mathrm{G}^{* *}$ except for molecule 1. DFT results show higher values than the other ab initio ones (HF). The difference obtained between the two DFT types is weak and thus, the influence of the functional choice is weak during the hyperpolarizability calculation. The electronic correlation resulting from the use of MP2 method, increased the first order hyperpolarizability of molecules 1,2 , and 5 by $46.06 \%, 68.71 \%$, and of $22.07 \%$ respectively compared to the HF method. The results show also that MP2 values are the most higher. Further, it is surprising that the inclusion of electron correlation is required to obtain the correct tendency of first hyperpolarizability $\beta$.

We conclude that the electronic correlation has a crucial role in the first hyperpolarizability determination. Moreover various theoretical results PM3, HF, DFT and MP2 shows that the hyperpolarizability is increased with the increase in the attraction force of the acceptors-donors groups (Figure 2). Several theoretical and experimental studies have focused their attention on the understanding and design of new chromophores with large quadratic NLO properties such as azoic compounds. [30-38]

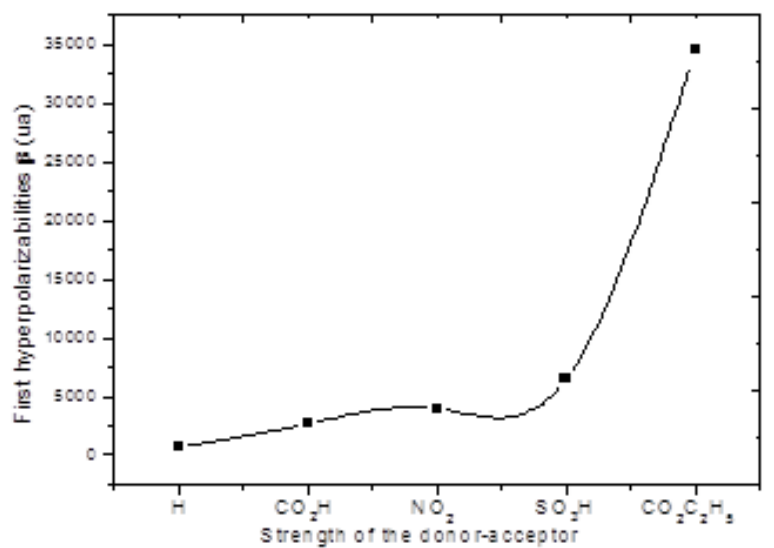

Figure 2. Variation of first hyperpolarizabilities $\beta$ with strength of the donor-acceptor groups

Several factors influence the electrons distribution and the nature of the HOMO and LUMO orbital. Some of these factors are noncentrosymetry, molecular geometry and the forces of the donor and acceptor electron groups. As a model we studied the dihedral angle between the $\mathrm{R}$ group and the phenyl (Figure 3) variation effect on the energies of the HOMO and LUMO orbitals, the HOMO-LUMO energetic gap and the hyperpolarizability using PM3 method for the molecules 3 and 5 .

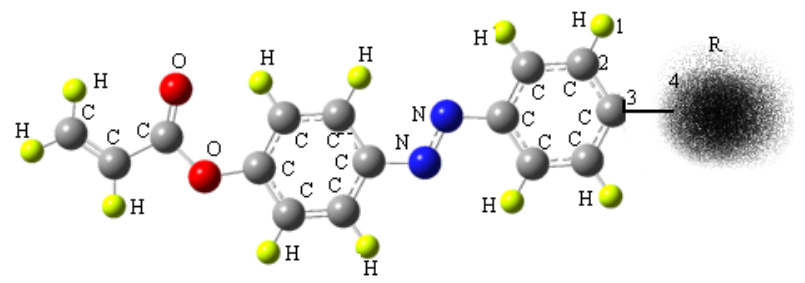

Figure 3. $\Phi=\mathrm{R}-\mathrm{C}_{3}-\mathrm{C}_{2}-\mathrm{H}_{1}$ dihedral angle between the phenyl and the substituted group $\mathrm{R}$ 
The results show that the hyperpolarizability is sensitive to geometrical variation as shown in Figure 4. The maximum of hyperpolarizability is obtained when $\mathrm{R}-\mathrm{C}_{3}-\mathrm{C}_{2}-\mathrm{H}_{1}$ dihedral angle is $120^{\circ}$ up to $180^{\circ}$, moreover we notice that the hyperpolarizability $\beta$ maximum is obtained when the molecule is plane. This last property is very important for this type of compounds.

Table 3: |HOMO-LUMO| energetic gap values in $\mathrm{eV}$ and hyperpolarizability $\beta$ in au according to the dihedral angle for molecule 3 with PM3 calculation

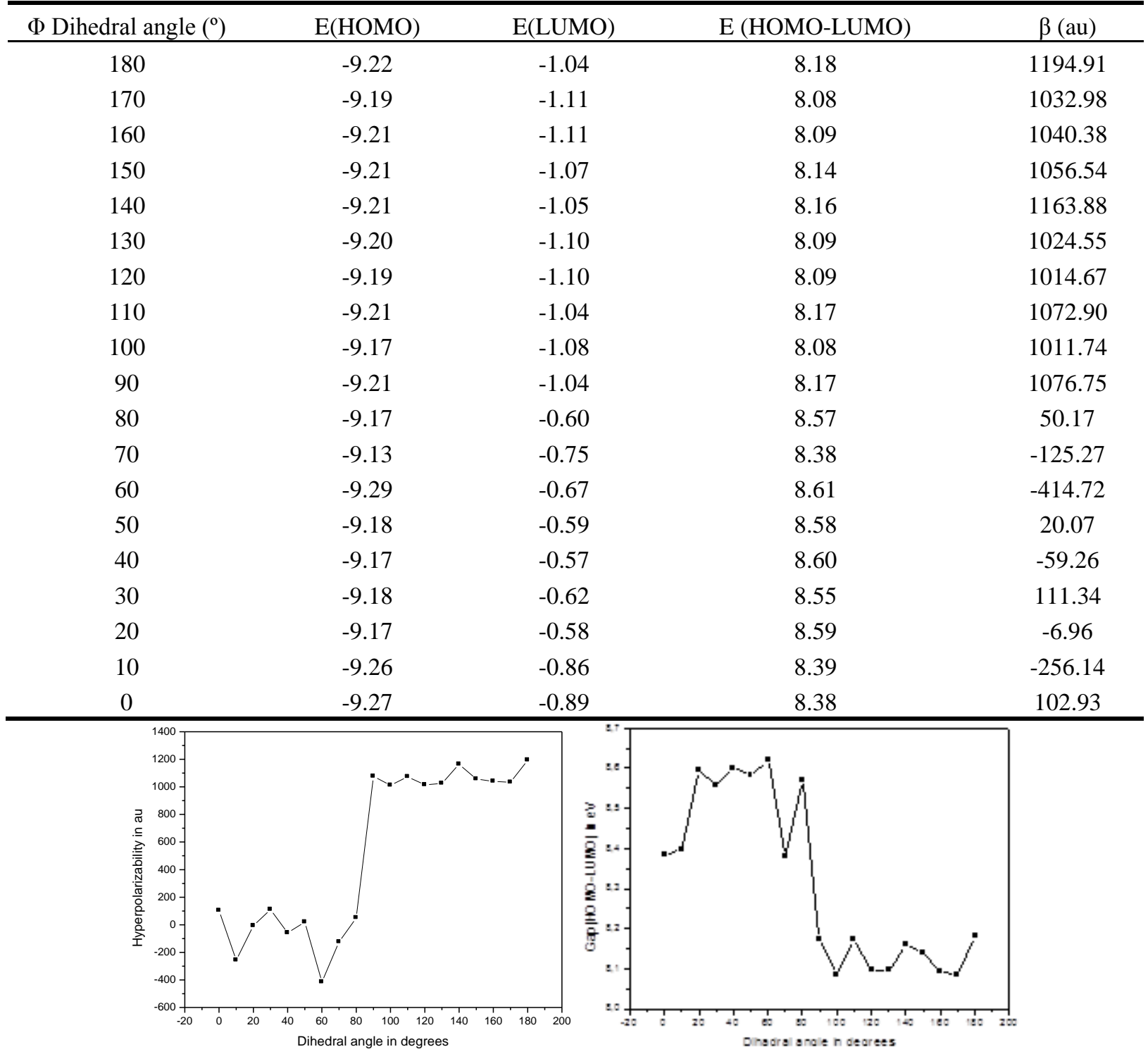

Figure 4. The variation of first hyperpolarizability and |HOMO-LUMO| energetic gap with the dihedral angle for molecule 3 with PM3 calculation

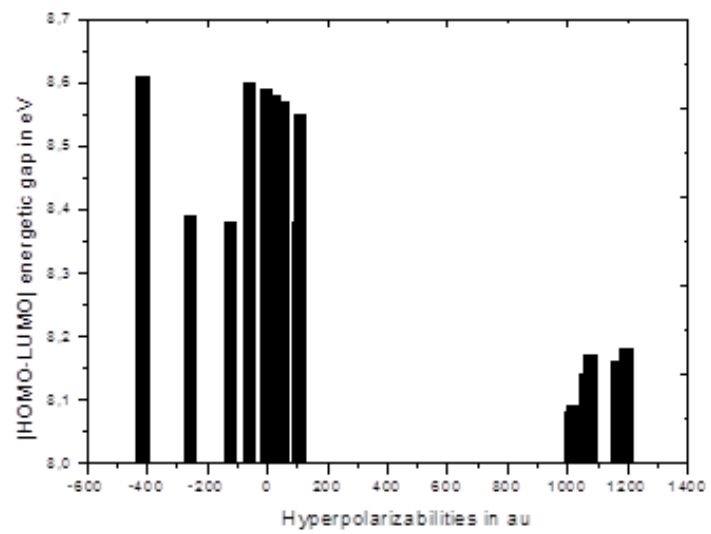

Figure 5. A plot of HOMO-LUMO gap versus hyperpolarizability for molecule 3 with PM3 calculation 
The variation of HOMO-LUMO energetic gap with the dihedral angle $\Phi$ (Figure 3 ) for molecules 3 and 5 are sketched in Figures 4 and 6. The general trend for all molecules is that the optimum dihedral angle for maximum hyperpolarizability would be $120^{\circ}-180^{\circ}$. This will clearly show that the maximum hyperpolarizability is obtained for a planar structure for all molecules. It is also seen from Figures 5 and 7 , there is an inverse relationship between hyperpolarizability and the HOMO-LUMO energetic gap.

In the same way, the hyperpolarizability, HOMO energy, LUMO energy and |HOMO-LUMO| energetic gap are very sensible to any geometrical variation.

The Figures 8 and 9 show the electron acceptor groups $\left(-\mathrm{CO}_{2} \mathrm{H},-\mathrm{CO}_{2} \mathrm{C}_{2} \mathrm{H}_{5}\right)$ participation to both LUMO and HOMO frontier orbitals. The HOMO orbital is delocalized over the whole $\mathrm{C}-\mathrm{N}$ bond of the spacer (although with a contribution from the vinyl ring linked to the acceptor smaller than that linked to the donor). We note from the LUMO frontier orbital, that the electronic density is concentrated on the cycles, and on the azo bridge $\mathrm{N}=\mathrm{N}$.

Table 4: |HOMO-LUMO| energetic gap values in eV and hyperpolarizability $\beta$ in au according to the dihedral angle for molecule 5 with PM3 calculation

\begin{tabular}{ccccc}
\hline$\Phi$ Dihedral angle $\left(^{\circ}\right)$ & E(HOMO) & E(LUMO) & E|HOMO-LUMO & $\beta(\mathrm{au})$ \\
\hline 180 & -8.49 & -2.10 & 6.39 & 1138.45 \\
170 & -8.49 & -2.08 & 6.40 & 1118.77 \\
160 & -8.47 & -2.06 & 6.41 & 1091.64 \\
150 & -7.68 & -1.99 & 6.41 & 1119.23 \\
140 & -7.68 & -1.15 & 6.52 & 872.71 \\
130 & -7.75 & -1.11 & 6.64 & 967.82 \\
120 & -8.08 & -1.15 & 6.93 & 990.42 \\
110 & -8.17 & -1.17 & 7.00 & 901.95 \\
100 & -8.20 & -1.14 & 7.03 & 918.29 \\
90 & -8.24 & -1.13 & 7.10 & 904.70 \\
80 & -8.54 & -1.15 & 7.38 & 863.56 \\
70 & -8.67 & -1.16 & 7.51 & 725.09 \\
60 & -8.67 & -1.04 & 7.71 & 786.49 \\
50 & -9.00 & -1.18 & 7.82 & 820.91 \\
40 & -9.17 & -1.17 & 8.00 & 697.92 \\
30 & -9.27 & -1.16 & 8.11 & 712.18 \\
20 & -9.32 & -1.17 & 8.15 & 588.34 \\
10 & -9.35 & -1.17 & 8.17 & 644.04 \\
0 & -9.37 & -1.18 & 8.19 & 631.01 \\
\hline
\end{tabular}
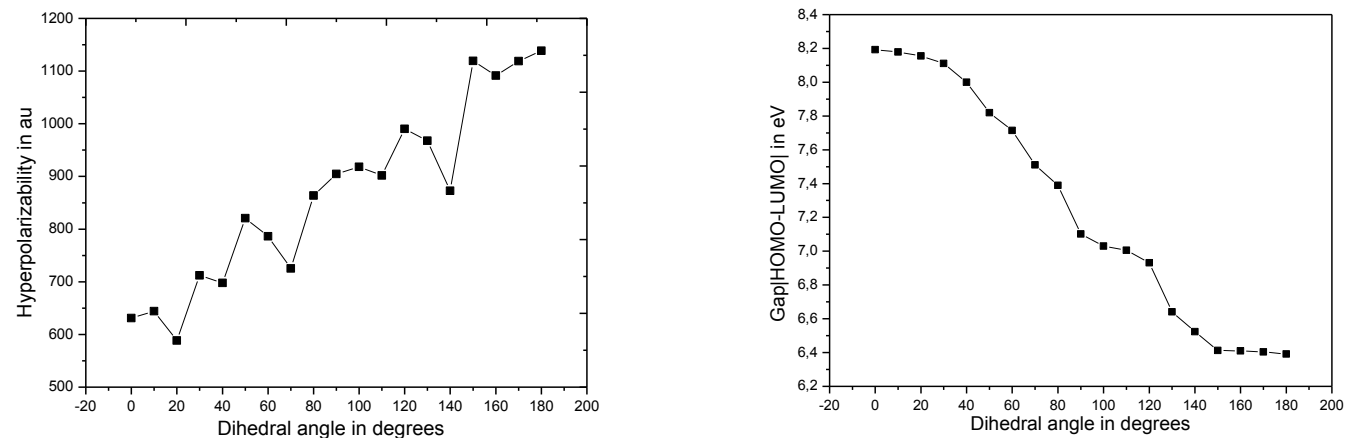

Figure 6. The variation of first hyperpolarizability and |HOMO-LUMO| energetic gap with the dihedral angle for molecule 5 with PM3 calculation 


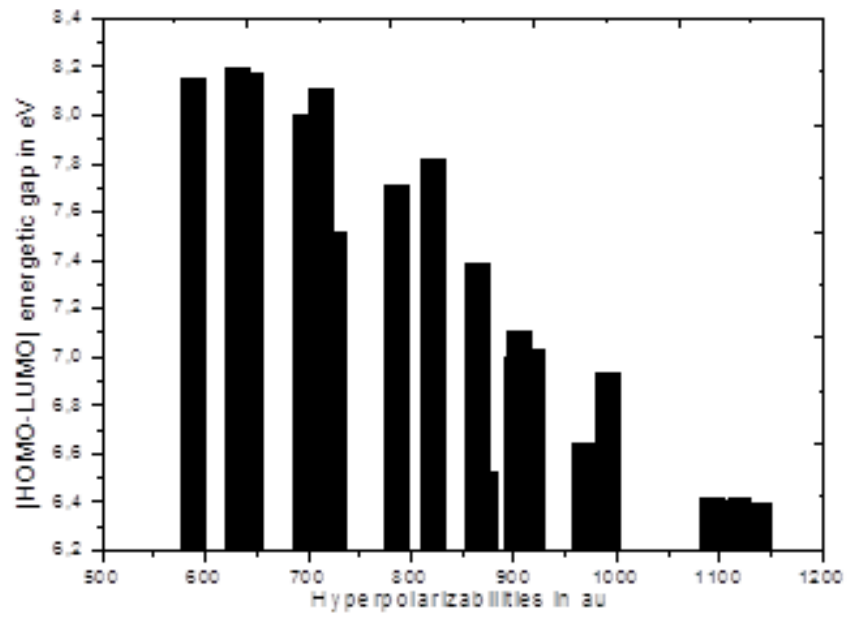

Figure 7. A plot of HOMO-LUMO gap versus hyperpolarizability for molecule 5 with PM3 calculation

The HOMO and LUMO representations in Figures 8 and 9, allowed us to appreciate the influence of the substituted groups on the electronic distribution which characterizes the generating area of electronic density, and to see the charge transfer density between the groups and the rest of the molecule. Consequently, the HOMO-LUMO transition implies an electron density transfer from the more aromatic part of the $\pi$-conjugated spacer including the electron donor group. The HOMO represents the ability to donate an electron, the LUMO instead, is an electron acceptor with the ability to obtain an electron. Moreover, these two molecular orbitals significantly overlap in the middle region of the electron density, which is an inherent condition to achieve an efficient charge transfer and, consequently, the nonlinear optical response of push-pull NLO chromophores.
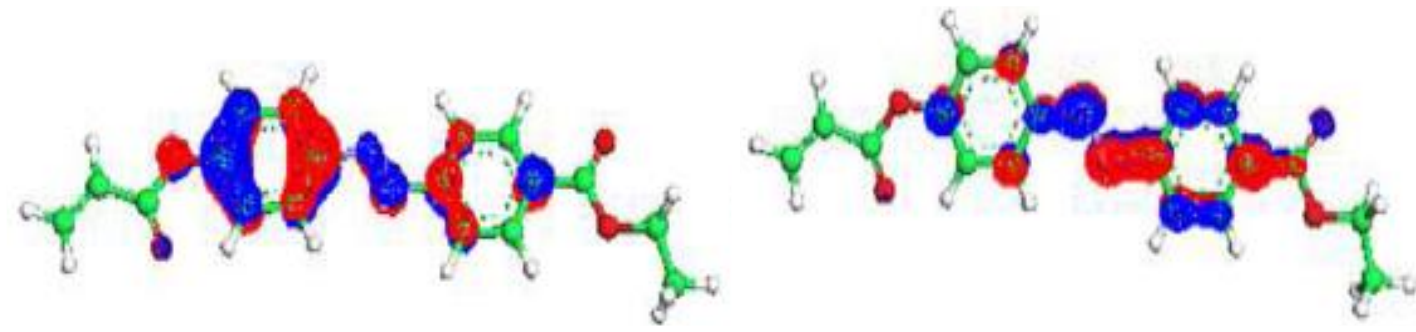

Figure 8. Representations of the HOMO frontier orbital (right) and LUMO frontier orbital (left) for molecule 3 with PM3 calculation

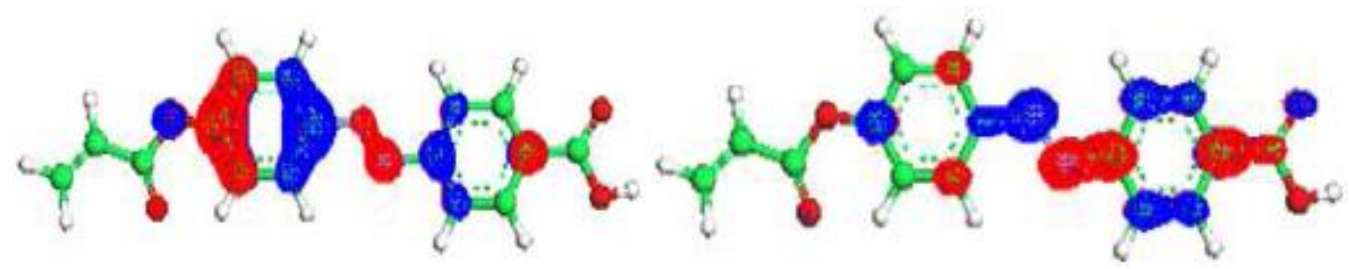

Figure 9. Representations of the HOMO frontier orbital (right) and LUMO frontier orbital (left) for molecule 5 with PM3 calculation

\section{Conclusion}

Important observations of general interest in this theoretical study of NLO proprieties of these azoics dyes are presented in this work. It is seen that different methods, semiempirical, Hartree-Fock, Møller-Plesset perturbation theory and density functional, all give relatively consistent descriptions of the electronic properties for different molecules having widely different properties.

High hyperpolarizability values were obtained by modification of the electron-acceptor groups. We obtained with these numerical simulations reliable information on the geometrical structure role and on the subsistent variation of the NLO properties. The theoretical calculation seems to be more helpful to determine particular components of hyperpolarizability tensor than to establish the real values of hyperpolarizability. They constitute an invaluable tool to predict the evolution of the molecular characteristics and allow thus to optimize the molecule choice before the synthesis. It is obvious that the first hyperpolarizability is strongly dependent on the extent of the electronic communication between the group and through the bridge (the softening of the $\mathrm{N}=\mathrm{N}$ bond participates in the transfer of electron density from the donor to the acceptor.) We have also observed that the 
hyperpolarizability is dependent on the molecular geometry.

The highest occupied molecular orbital (HOMO) and the lowest unoccupied molecular orbital (LUMO) examination shows that the first hyperpolarizability of these derivatives is directly related to the HOMO-LUMO energetic gap with an inverse relationship.

This study reveals that these molecular systems have very large first hyperpolarizability and should be excellent candidates to further experimentation.

\section{References}

1 - F. T. Arecchi, E. O. Schulz-Dubois, Laser Handbook, North Holland, Amsterdam, 1972.

2 - L. R. Dalton, A. W. Harper, R. Ghosn, W. H. Steier, M. Ziari, H. Fetterman, Y. Shi, R. V. Mustacich, A. Jen, K. Y. Shea. Chem. Mater, 1995, 7, 1060.

3 - D. M. Burland, R. D. Miller, C. A. Walsh. Chem. Rev, 1994, 94, 31.

4 - T. H. Maiman, Nature, 1960, 189, 493.

5 - P. A. Franken, C.W. Peters, G. Weinreich, Phys. Rev, 1961, 7, 118.

6 - D. J. Williamsm, American Chemical Society, Washington DC, 1985.

7 - D. S. Chemla, J. Zyss, Academic Press, Orlando, 1987.

8 - R. A. Hann, D. Bloorm, The Royal Society of Chemistry, Cambridge, 1991.

9 - J. Zyss, Academic Press, Boston, 1994.

10 - T. Kobayashim, Springer, Berlin, 1989.

11 - P. N. Prasad, D. J. Williams, Introduction to Nonlinear Optical Effects in Molecules and Polymers, Wiley, New York, 1990.

12 - D. R. Kanis, M. A. Ratner, T. J. Marks, Chem. Rev, 1994, 94, 195.

13 - W. Bartkowiak, T. Misiaszek, Chem. Phys, 2000, 261, 353.

14 - L. J. Rao, K. Bhanuprakash, Indian. J. Chem, 2000, 39, 114.

15 - J. Lipinski, W. Bartkowiak, Chem. Phys, 1999, 245, 263.

16 - T. R. Cundari, H. Z. Kurtz, T. Zhou, J. Phys. Chem, 2000, 104, 4711.

17 - S. Brasselet, J. Zyss, J. Opt. Soc. Am. B, 1998, $15,257$.
18 - B. H. Cardelino, C. E. Morre, R. E. Stickel, J. Phys. Chem, 1991, 95, 8645.

19 - M. J. Frisch et al, 2003.

20 - C. Dehu, F. Meyers, E. Hendrickx, K. Clays, A. Parsons, S. R. Marder, J. L. Bredes, J. Am. Chem. Soc, 1995, 117, 10127.

21 - F. Meyers, J. Zyss, J. Am. Chem. Soc, 1992, 114, 2914.

22 - D. A. Kleinman, Phys. Rev, 1962, 126, 1977.

23 - H. Hashimoto, T. Nakashima, K. Hattori, T. Yamada, T. Mizoguchi, Y. Koyama, T. Kobayashi, Pure, Appl. Chem, 1999, 71, 12.

24 - J. J. P. Stewart, J. Comput. Chem, 1989, 10, 209.

25 - H. Durr, H. B Laurent. Amsterdam, Elsevier, 1990.

26 - A. V. Epstov. Organic photochromes, New York, Plenum Press, 1971.

27 - M. Korbus , G. Balasubramanian , F. M. Plathe, H. Kolmar, M. F. J Almes, 2014, 395, 401-12.

28 - R. L. Sutherland. Handbook of nonlinear optics. New York, Marcer Dekker, 1996.

29 - Z.Sekkat, W. Knoll. Photoreactive organic thin films. Amsterdam: Academic Press, 2002.

30 - K. Traskovskis, E. Zarins, L. Laipniece, A. Tokmakovs, V. Kokars, M. Rutkis, Materials Chemistry and Physics, 2015, 155, 232-240.

31 - M. C. Spiridon, F. A. Jerca, V. V. Jerca, D. M. Vuluga, D. S. Vasilescu; U. P. B. Sci. Bull, 2014, 76, 1.

32 - N. Islam, and A. H. Pandith, Int. J. Chem, 2014, $3,2$.

33 - S. N. Derrar, M. S. Rahal, M. Springborg, Journal of Physic, 2015, 574, 012007.

34 - F. Ye, F. Qiu, D. Yang, G. Cao, Y. Guan, L. Zhuang, Optics \& Laser Technology, 2013, 49, 56-63.

35 - M. C. Spiridon, K. Iliopoulos, F. A. Jerca, V. V. Jerca, D. M. Vuluga, D. S. Vasilescu, Dyes and Pigments, 2015, 114, 24-32.

36 - F. Zhao, C. Wang, M. Qin, P. Zeng, P. Cai, Optics Communications, 2015, 338, 461-466.

37 - R. D. Breukers, S. Janssens, S. G. Raymond, M. D. H. Bhuiyan, A. J. Kay, Dyes and Pigments, 2015, 112, 17-23.

38 - C. Constantinescu, A. Matei, I. Ionita, V. Ion, V. Marascu, M. Dinescu, C. Vasiliuc, A. Emandi, Applied Surface Science, 2014, 302, 69-73. 